\title{
Differential expression of the RNA Polymerase III polypeptide K, POLR3K, in cancers of
} the breast.

Shahan Mamoor, MS1

1shahanmamoor@gmail.com

East Islip, NY 11730

Breast cancer affects women at relatively high frequency ${ }^{1}$. We mined published microarray datasets 2,3 to determine in an unbiased fashion and at the systems level genes most differentially expressed in the primary tumors of patients with breast cancer. We report here significant differential expression of the gene encoding the RNA polymerase III polypeptide K, POLR3K, when comparing primary tumors of the breast to the tissue of origin, the normal breast. While expression of POLR3K in primary tumors of the breast was significantly higher than in normal breast tissue, distant metastasis-free survival in lymph node positive patients was significantly longer in patients with high tumor expression of POLR3K than in patients with low tumor expression of POLR3K. POLR3K may be of relevance to initiation, maintenance or progression of cancers of the female breast.

Keywords: breast cancer, POLR3K, RNA Polymerase III polypeptide K, systems biology of breast cancer, targeted therapeutics in breast cancer. 
Invasive breast cancer is diagnosed in over a quarter of a million women in the United States each year ${ }^{1}$ and in 2018 , breast cancer was the leading cause of cancer death in women worldwide ${ }^{4}$. While patients with localized breast cancer are provided a $99 \% 5$-year survival rate patients with regional breast cancer, cancer that has spread to lymph nodes or nearby structures, are provided an $86 \% 5$-year survival rate 5,6 . Patients with metastasis to distant sites are provided a $27 \% 5$-year survival rate 5,6 . Understanding how primary tumors are most transcriptionally different from the tissue from which they originate, the breast, can facilitate development of novel diagnostic and therapeutics to promote early detection and enhanced treatment, and contribute to efforts to prevent progression to metastatic stages. We mined published microarray data 2,3 to understand at the transcriptome level and in an unbiased fashion genes most differentially expressed in primary tumors of the breast as compared to normal breast tissue. The RNA polymerase III polypeptide $\mathrm{K}$ emerged as among the most differentially expressed genes in cancer of the female breast.

\section{Methods}

We utilized datasets GSE $109169^{2}$ and GSE124648³ for this differential gene expression analysis of female breast cancer. GSE109169 was generated using Affymetrix Human Exon 1.0 ST Array technology with $n=25$ normal breast tissue and $n=25$ tumors of the breast and; analysis was performed using platform GPL5175. The tissues from this first dataset are paired tissues (25 tumors matching 25 breast tissues from 25 patients). GSE124648 was generated using Affymetrix Human Genome U133A Array technology with $n=10$ normal breast tissue and $n=126$ tumors of the breast and; analysis was performed using platform GPL96. The Benjamini and Hochberg method of $p$-value adjustment was used for ranking of differential 
expression but raw $p$-values were used to assess statistical significance of global differential expression. Log-transformation of data was auto-detected, and the NCBI generated category of platform annotation was used. A statistical test was performed to evaluate whether POLR3K expression was significantly between primary breast tumors and breast tissue using a twotailed, unpaired t-test with Welch's correction. We used PRISM for all statistical analyses of differential gene expression in human breast cancer (Version 8.4.0)(455). For Kaplan-Meier survival analysis, we used the Kaplan-Meier plotter online tool7 for correlation of POLR3K mRNA expression levels with distant metastasis-free survival (DMFS) in $n=382$ breast cancer patients with lymph node positive cancer in the lower survival quartile.

\section{Results}

We performed discovery of genes associated with breast cancer in females by mining two independently published microarray datasets 2,3 .

\section{POLR3K is differentially expressed in primary tumors of the breast.}

Comparison of 25 normal breast tissues to 25 tumors of the breast ${ }^{2}$ revealed that the gene encoding the RNA polymerase polypeptide $\mathrm{K}$, encoded by the POLR3K gene, was among the genes most differentially expressed in tumors of the breast in female breast cancer (Table 1). When sorting each of the genes expressed in tumors of the breast based on significance of difference as compared to normal breast tissue, POLR3K ranked 101 out of 19076 total transcripts, equating to $99.5 \%$ differential expression (Table 1). Differential expression of POLR3K in female breast cancer was statistically significant (Table $1 ; p=1.26 \mathrm{E}-14$ ).

Analysis of a second microarray dataset ${ }^{3}$, here in 126 tumors of the breast as compared to 10 normal breast tissues, again revealed significant differential expression of POLR3K in 
tumors of the breast in female breast cancer (Table 2). When sorting each of the genes expressed in tumors of the breast based on significance of difference as compared to normal breast tissue, POLR3K ranked 418 out of 22283 total transcripts, equating to $98.1 \%$ differential expression (Table 2). Differential expression of POLR3K in female breast cancer was statistically significant (Table $2 ; p=1.26 \mathrm{E}-11$ ).

POLR3K is expressed at significantly higher levels in breast tumors as compared to the breast.

We obtained exact mRNA expression levels for POLR3K from the breast and from breast tumors to understand the magnitude and direction of POLR3K expression change. In both datasets, POLR3K was expressed at higher levels in tumors of the breast as compared to normal breast tissue (Figure 1). Increased expression of POLR3K in primary breast tumors was statistically significant (Figure 1: $p<0.0001$ ). We calculated a mean fold change of $1.10 \pm 0.03$ when comparing primary tumors of the breast to normal breast tissues (Table 1).

POLR3K expression associates with survival outcomes in lymph node positive breast cancer.

We performed Kaplan-Meier survival analysis in an attempt to correlate POLR3K mRNA expression levels with survival outcomes in 382 lymph node positive breast cancer patients. We found a statistically significant correlation between POLR3K expression and distant metastasis-free survival (DMFS) in patients with breast cancer (Figure 2; log rank $p$-value: 0.045 for distant metastasis-free survival, hazard ratio: 0.65 (0.42-0.99) (Fig. 2)).

Lower POLR3K mRNA levels were a negative prognostic indicator in lymph node positive 
patients. Median DMFS was 24 months for lymph node positive patients with low tumor expression of POLR3K while median RFS was 68.71 months for patients with high tumor expression of POLR3K (Table 3).

Thus, through comparative transcriptome analysis of primary tumors of the breast and normal breast tissue, we found that differential expression and up-regulation of POLR3K was among the most significant transcriptional features in primary tumors from patients with breast cancer. POLR3K expression in primary tumors of the breast demonstrated a strong and significant correlation with distant metastasis-free survival in patients with lymph node positive breast cancer in the lower survival quartile, with higher expression of POLR3K correlating with improved survival.

\section{Discussion}

Invasive breast cancer is medical problem with a 27\% 5-year survival rate for women whose disease has spread to distant sites. To facilitate understanding of the basic transcriptional differences between primary tumors of the breast and the tissues from which these tumors originate, normal breast tissues, we performed comparative transcriptome analysis using two independently published microarray datasets 2,3 and identified significant differential expression of POLR3K in breast cancer.

Transcription of cellular genes is performed by three major RNA polymerases. One of these is RNA polymerase III, which contains 17 subunits, including POLR3K. The yeast homolog of POLR3K, known as C11, displays homology to TFIIS, to A12.2 from RNA polymerase I, to B12.6 from RNA polymerase II, and is important for RNA cleavage during termination of transcription ${ }^{8}$. The carboxy-terminus of POLR3K shares sequences similarity with the carboxy-terminus of the RNA polymerase II elongation factor ${ }^{8}$. C11 is a protein of 12.5 
kilodaltons, contains a region with homology to the amino-terminal $Z n$ binding domain of Rpb9p, an RNA polymerase II subunit, as well as a region with homology to the $\mathrm{Zn}$ ribbon motif of TFIIS that functions in 3' RNA cleavage ${ }^{9}$. Broadly, transcription occurs in three major steps: initiation, elongation, and termination. It has been suggested that $\mathrm{C} 11$ functions to allow RNA polymerase III to switch between transcriptional elongation and cleavage of the nascent transcript ${ }^{8}$.

Evidence of such a role for $\mathrm{C} 11$ is that an RNA polymerase III mutant purified from yeast that do not contain $\mathrm{C} 11$ can function as wild-type in initiation of transcription but fails to recognize pause sites and is impaired in transcription termination ${ }^{8}$. Of relevance here is the existence of a termination signal that provides information to the RNA polymerase with respect to when transcriptional termination should be executed. The termination signal for RNA polymerase III is a stretch of thymines known as an oligo $\mathrm{d}(\mathrm{T})$ tract, or oligo $\mathrm{d}(\mathrm{T})$ "terminator"; RNA polymerase III pauses at this terminator, and termination is coupled with release of the nascent transcript by cleavage. A mutation in C11, C11-F32S, results in inability of RNA polymerase to pause at oligo $d(T)$ tracts, terminators $d T(6)$ and $d T(7) ; F 32$ resides in an amino-terminal Rpb9 homologous domain of $\mathrm{C} 11^{9}$. Another mutant of $\mathrm{C} 11, \mathrm{C} 11-\mathrm{C} 102 \mathrm{~S}$, possesses approximately $20 \%$ of RNA 3'-5' cleavage activity of wild-type C11. Thus, it appears that C11, and by homology, the human gene product of POLR3K, possesses two transcription termination-related functions: 1) recognition of a termination site, the oligo $\mathrm{d}(\mathrm{T})$ tract, performed by the amino-terminus of $\mathrm{C} 11$; and 2) 3'-5' RNA cleavage to release the nascent RNA transcript during transcriptional termination, performed by the carboxy-terminus of C11 - the TFIIS homologous domain. A third potential function for C11 may be in the "recycling" of RNA Polymerase III during transcription, or reinitiation of transcription after transcription termination ${ }^{10}$.

A zebrafish mutant of polr3b is phenotypically defective in development of the digestive organs; the mutation, RNA polymerase III, when isolated from yeast that model this same 
mutation in POLR3B, contains reduced levels of POLR3K, suggesting that the gene products of POLR3B and POLR3K interact ${ }^{11}$. An R41W mutation was identified in 2 unrelated patients with hypomyelinating leukodystrophy, and fibroblasts from these patients display a $60-80 \%$ reduction in $5 \mathrm{~S}$ and $7 \mathrm{~S}$ ribosomal RNAs ${ }^{12}$. We could not identify literature describing a role for POLR3K in human breast cancer, but one study described over-expression of RNA Polymerase III K polypeptide in tumors from patients with pancreatic ductal adenocarcinoma (PDAC), and depletion of the POLR3K gene product in three separated PDAC cell lines resulted in reduced cell proliferation, indicating that in the context of at least one human cancer, RNA polymerase III polypeptide K could support cell proliferation. Reduction of POLR3K also impaired anchorageindependent cell growth ${ }^{13}$.

We report here differential expression of the RNA Polymerase III polypeptide K, encoded by the POLR3K gene in human breast cancer. POLR3K was expressed at significantly higher levels in primary tumors from patients with breast cancer, and in lymph node positive breast cancer patients, high expression of POLR3K was associated with superior distant metastasisfree survival in patients of the lower survival quartile. RNA polymerase III polypeptide $\mathrm{K}$, and the processes to which it pertains to during transcriptional termination, may be relevant to the initiation or progression of human breast cancer.

7 OF 14 


\section{References}

1. DeSantis, C.E., Ma, J., Goding Sauer, A., Newman, L.A. and Jemal, A., 2017. Breast cancer statistics, 2017, racial disparity in mortality by state. CA: a cancer journal for clinicians, 67(6), pp.439-448.

2. Chang, J.W., Kuo, W.H., Lin, C.M., Chen, W.L., Chan, S.H., Chiu, M.F., Chang, I.S., Jiang, S.S., Tsai, F.Y., Chen, C.H. and Huang, P.H., 2018. Wild-type p53 upregulates an early onset breast cancer-associated gene GAS7 to suppress metastasis via GAS7-CYFIP1mediated signaling pathway. Oncogene, 37(30), pp.4137-4150.

3. Sinn, B.V., Fu, C., Lau, R., Litton, J., Tsai, T.H., Murthy, R., Tam, A., Andreopoulou, E., Gong, Y., Murthy, R. and Gould, R., 2019. SET ER/PR: a robust 18-gene predictor for sensitivity to endocrine therapy for metastatic breast cancer. NPJ breast cancer, 5(1), pp. $1-8$.

4. Bray, F., Ferlay, J., Soerjomataram, I., Siegel, R.L., Torre, L.A. and Jemal, A., 2018. Global cancer statistics 2018: GLOBOCAN estimates of incidence and mortality worldwide for 36 cancers in 185 countries. CA: a cancer journal for clinicians, 68(6), pp.394-424.

5. ACS Cancer Facts \& Figures 2019. https://www.cancer.net/cancer-types/breast-cancermetastatic/statistics.

6. Survival Rates for Breast Cancer. https://www.cancer.org/cancer/breast-cancer/ understanding-a-breast-cancer-diagnosis/breast-cancer-survival-rates.html

7. Györffy, B., Lanczky, A., Eklund, A.C., Denkert, C., Budczies, J., Li, Q. and Szallasi, Z., 2010. An online survival analysis tool to rapidly assess the effect of 22,277 genes on breast cancer prognosis using microarray data of 1,809 patients. Breast cancer research and treatment, 123(3), pp.725-731.

8. Chédin, S., Riva, M., Schultz, P., Sentenac, A. and Carles, C., 1998. The RNA cleavage activity of RNA polymerase III is mediated by an essential TFIIS-like subunit and is important for transcription termination. Genes \& development, 12(24), pp.3857-3871.

9. Iben, J.R., Mazeika, J.K., Hasson, S., Rijal, K., Arimbasseri, A.G., Russo, A.N. and Maraia, R.J., 2011. Point mutations in the Rpb9-homologous domain of Rpc11 that impair transcription termination by RNA polymerase III. Nucleic acids research, 39(14), pp. 6100-6113. 
10. Landrieux, E., Alic, N., Ducrot, C., Acker, J., Riva, M. and Carles, C., 2006. A subcomplex of RNA polymerase III subunits involved in transcription termination and reinitiation. The EMBO journal, 25(1), pp.118-128.

11. Yee, N.S., Gong, W., Huang, Y., Lorent, K., Dolan, A.C., Maraia, R.J. and Pack, M., 2007. Mutation of RNA Pol III subunit rpc2/polr3b Leads to Deficiency of Subunit Rpc11 and disrupts zebrafish digestive development. PLoS Biol, 5(11), p.e312.

12. Dorboz, I., Dumay-Odelot, H., Boussaid, K., Bouyacoub, Y., Barreau, P., Samaan, S., Jmel, H., Eymard-Pierre, E., Cances, C., Bar, C. and Poulat, A.L., 2018. Mutation in POLR3K causes hypomyelinating leukodystrophy and abnormal ribosomal RNA regulation. Neurology Genetics, 4(6), p.e289.

13. Yang, Y., Schmidt, H., Kopp, T., Eick, L., Gress, T. and Buchholz, M., 2019. RNA polymerase III subunit $\mathrm{K}$ is overexpressed and regulates cell proliferation in Pancreatic Ductal Adenocarcinoma. Zeitschrift für Gastroenterologie, 57(09), pp.KV-325. 


\begin{tabular}{|c|c|c|c|c|c|c|}
\hline Rank & ID & p-value & $\mathbf{t}$ & B & Gene & Gene name \\
\hline 418 & 218866_s_at & $1.26 \mathrm{E}-11$ & 7.39815 & 16.04658 & POLR3K & $\begin{array}{l}\text { RNA polymerase III } \\
\text { subunit K }\end{array}$ \\
\hline
\end{tabular}

Table 2: POLR3K is differentially expressed in human breast cancer.

Rank of differential expression, probe ID, p-value of global differential expression, $t, a$ moderated t-statistic, B, the log-odds of differential expression between the groups compared, fold change of POLR3K in primary breast tumors as compared to normal breast, gene and gene name are listed in this chart. 


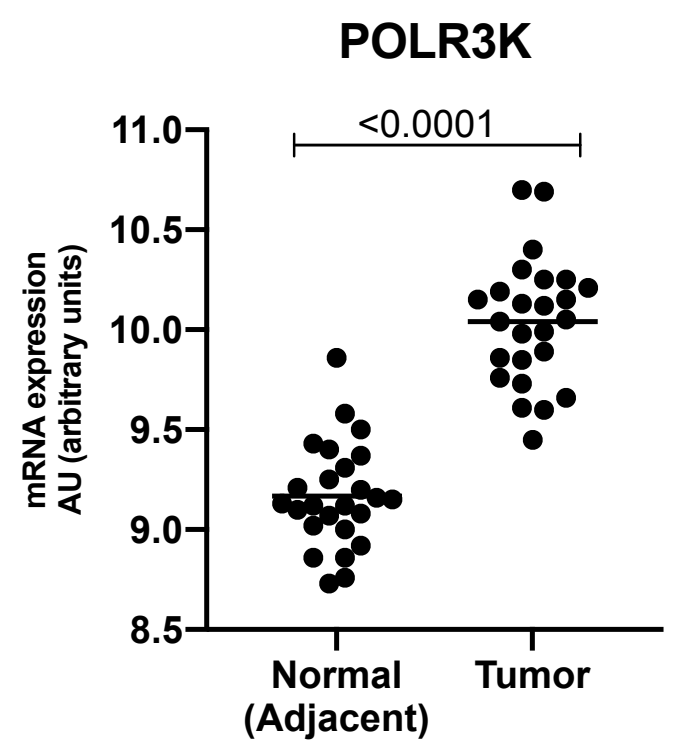

Figure 1: POLR3K is expressed at significantly higher levels in primary breast tumors as compared to normal breast tissue.

The mRNA expression level of POLR3K in normal breast tissue (left) and in primary tumors of the breast (right) is graphically depicted with the result of a statistical test evaluating significance of difference in POLR3K expression between normal breast tissue and primary tumors of the breast, a $p$-value, listed above. 


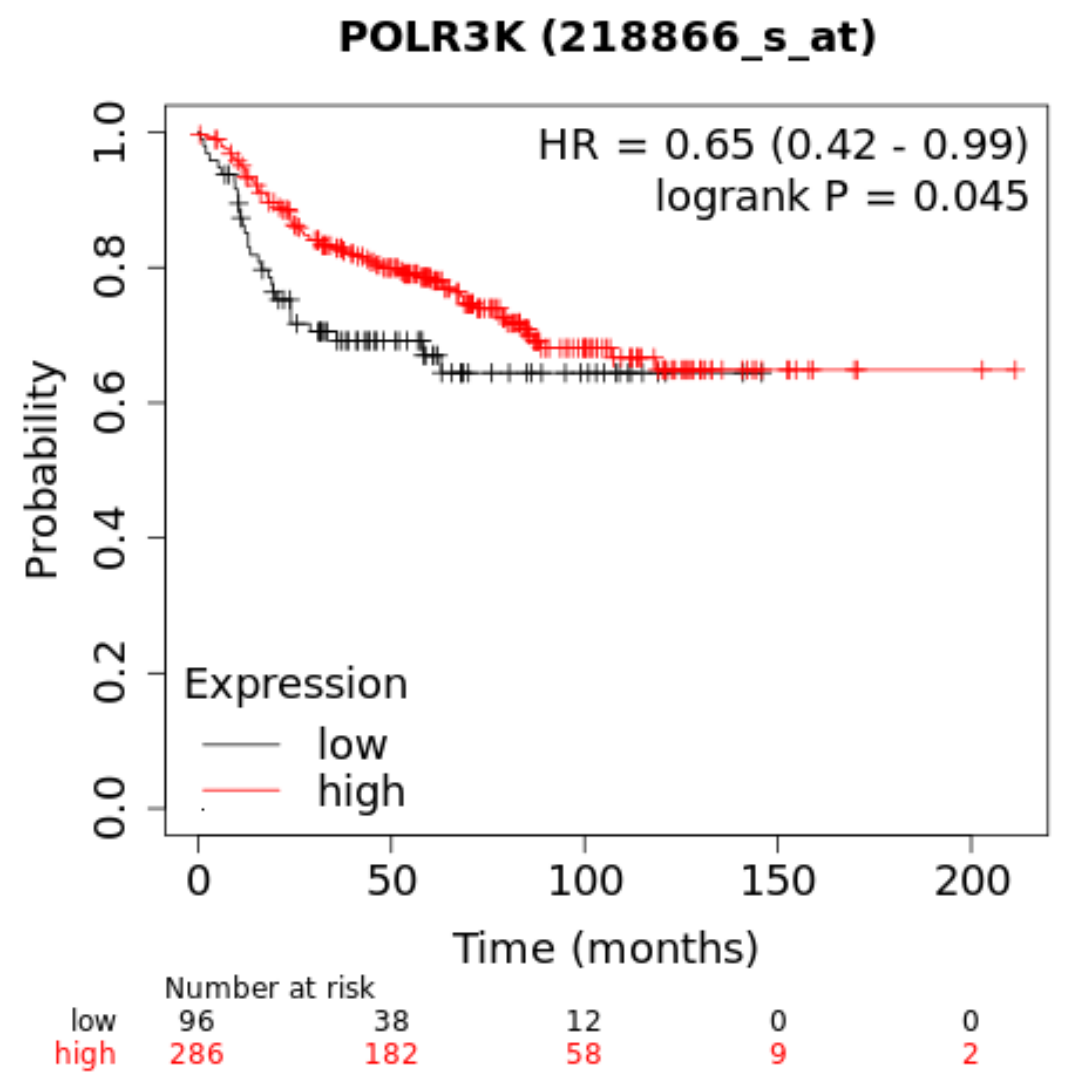

Figure 2: POLR3K expression correlates with distant metastasis-free survival in patients with lymph node positive breast cancer.

Depicted in this Kaplan-Meier plot is the probability of distant metastasis-free survival (DMFS) for $n=382$ total patients stratified into two groups, based on low or high expression of POLR3K in patient primary tumors, in the lower survival quartile. The log rank $p$-value denoting statistical significance of difference in distant metastasis-free survival when comparing the two groups, as well as hazard ratio for this comparison is listed above. Listed below is the number of patients at risk (number of patients alive) per interval, after stratification based on POLR3K expression; in the first interval, number at risk is number of patients alive; in each subsequent interval, number at risk is the number at risk less those who have expired or are censored. 

in lymph node positive breast cancer patients with high primary tumor expression of POLR3K.

The relapse-free survival of $n=382$ lymph node positive breast cancer patients based on stratification into low or high tumor expression of POLR3K is listed in this chart, for patients in the lower survival quartile.

$$
24
$$

68.71

High expression cohort (months) 